\title{
Reducing Drag and Oscillation of Spheres Used for Buoyancy in Oceanographic Moorings
}

\author{
Jane Hurley and Brad de Young \\ Department of Physics and Physical Oceanography, Memorial University of Newfoundland, St. John's, Newfoundland, Canada \\ Christopher D. WiLliams \\ National Research Council, Canada Institute for Ocean Technology, St. John's, Newfoundland, Canada
}

(Manuscript received 21 December 2005, in final form 7 October 2006)

\begin{abstract}
Numerical and laboratory results of the drag characteristics are presented for different configurations of an underwater buoyancy package. It is shown that the drag and oscillation of an underwater sphere can be reduced substantially with the addition of a shaped cowling. The influence of several different cowling shapes on the drag and lift are determined. The results from a numerical fluid dynamical calculation are compared and laboratory measurements are scaled. Both the dynamic and static components of drag and lift are presented. The drag force for an underwater sphere can be reduced by more than $80 \%$ for a full teardrop-shaped cowling. A truncated teardrop, more practical for real applications, will still reduce the drag by $60 \%-70 \%$. In addition to the drag, the amplitude of oscillations driven by eddy shedding is similarly reduced.
\end{abstract}

\section{Introduction}

\section{a. Problem}

Drag on mooring components is one of the key constraints on the operational functionality of oceanographic moorings. The relative importance of drag from different mooring components depends upon the specific characteristics of each mooring (Berteaux 1991). Drag causes two primary problems-the instruments move deeper than deployed and change their position as the water velocity changes. A more subtle problem is the vibration induced in the mooring line by vortex shedding (Hamilton 2000), which is much like the strings on a musical instrument vibrating in response to being plucked. Vibrations in moorings have long been recognized as being a significant source of error (Hamilton et al. 1997).

Our goal is to determine how to reduce the effects of drag and oscillations on spherical buoys. Spherical buoys are commonly used because of their great

Corresponding author address: Brad de Young, Department of Physics, Memorial University of Newfoundland, St. John's, NL A1B 3X7, Canada.

E-mail: bdeyoung@physics.mun.ca strength for weight, their ease of construction, and their common availability. In this application, we wanted to maximize buoyancy but minimize drag in a mooring used in association with an underwater winch system (deYoung et al. 2005) deployed in shallow water.

The mooring-buoy system that we deploy consists of a subsurface spherical buoy of $0.7-\mathrm{m}$ diameter, which is anchored to the seafloor in shallow water $(\sim 20-\mathrm{m}$ depth) by a mooring line. An instrument package, consisting of two conductivity-temperature-depth (CTD) probes and several other instruments, is hung below the spherical buoy. The instrument package is connected with an electromechanical cable. An underwater winch is used to enable the package to profile from near the bottom to the surface, a distance of roughly $20 \mathrm{~m}$ (deYoung et al. 2005). The mooring site has strong tidal currents up to $1.0 \mathrm{~m} \mathrm{~s}^{-1}$ that cause the float to tilt and oscillate, thus limiting the vertical range of profiling.

We designed a cowling to cover the buoy that would reduce both the drag and the vortex shedding from the body. We tested the design through (a) preliminary computational fluid dynamics (CFD) analysis of seven streamlined shapes and confirmed these estimates by (b) scaled model experiments in a water tunnel on the two most promising shapes. 


\section{b. Background}

The drag force is a resistive force that opposes the motion of an object through a fluid, or the flow of a fluid about an object (Hoerner 1965). The total drag force on a submersed body is given by

$$
D_{B}=\frac{1}{2} \rho C_{D} A_{N} v^{2}
$$

where $\rho$ is the density of the fluid, and $C_{D}$ is a nondimensional drag coefficient that is dependent upon the body shape, the free-stream velocity as related by the Reynolds number ( $\mathrm{Re}$ ), the roughness of the surface of the body, and the turbulence level of the surrounding flow. In this study, $A_{N}$ is the frontal area of the body (cross-sectional area normal to the current flow) and $v$ is the free-streaming velocity of the flow. Quite often, the drag force is referred to in terms of the drag coefficient alone.

When fluid flows past a bluff object at a sufficiently high speed, the wake behind the object is no longer laminar. Rather, bluff objects experience separated flow over a large proportion of the body surface and, depending on the velocity of the impingent current, the wake varies in width and vortex structure (Berteaux 1991).

As a fluid particle flows toward the leading edge of a bluff body, the pressure in the fluid particle rises from the free-stream pressure to the stagnation pressure. The high fluid pressure near the leading edge impels flow around the bluff body as boundary layers develop on either side of a 2D body, and aft of the leading edge, as the flow approaches the widest portion of the body, the pressure can become negative.

At high Reynolds numbers, however, if there is insufficient streamwise momentum in the boundary layer to overcome this adverse pressure gradient, then near the widest section of the body the boundary layers separate from each side of the 2D surface and form two shear layers that travel in the direction of the flow, bounding the wake. Because the innermost portion of each shear layer, in contact with the bluff body, moves much more slowly (resulting from surface friction) than the outermost portion of the shear layers (resulting from contact with the free flow), the two shear layers roll into the near wake, where they fold upon each other, forming discrete swirling vortices (McGothlin 1982).

Vortex shedding is a periodic process with a frequency that is proportional to $v / d$, where $d$ is the width of the 2D bluff body measured perpendicular to the flow and $v$ is the incoming flow speed, such that the shedding frequency of a pair of alternating vortices to be produced is

$$
f_{s}=S(v / d),
$$

where $S$ is the Strouhal number, which is about 0.20 for a 2D circular cylinder and, for a sphere, is nearly constant at 0.22 for Reynolds numbers in the range being considered here: $500<\operatorname{Re}<100000$ (Brucker 2001).

Vortex shedding causes unsteady pressure loading on the body, resulting in a cyclic force acting on the body, typically sinusoidally with frequency $f_{s}$. This unsteady loading interacts with the body causing vortex-induced vibrations (VIVs), more commonly referred to as "strumming" when it occurs in either mooring lines or riser pipes. Because the flow is asymmetric fore and aft, as well as in the direction transverse to the flow, the body will experience time-varying forces in both the streamwise and cross-stream direction. For instance, in addition to a steady drag force, there are also fluctuating lift and drag forces associated with the individual shedding of vortices, because each individual shed vortex effectively "tugs" upon the body at the moment of detachment.

In the case of two-dimensional bluff bodies, such as circular cylinders, the drag force fluctuates at $2 f_{s}$, while the alternating lift force does so at $f_{s}$. Clearly, this is because the body will experience two tugs in the streamwise direction for each cycle of shedding, whereas the fluctuating lift force, which depends on a complete cycle of shedding, experiences only one such tug per period.

Much less experimental data exist for the wake of truly three-dimensional bluff bodies, such as axisymmetric bodies like the sphere. Such wake structures are truly three-dimensional because the vortices are now shed not only from fluid flow around the streamwise plane of the body, but from the cross-stream plane as well, and become very unsteady. Given the truly threedimensional nature of the wake, the drag, lift, and side forces are all expected to oscillate close to $f_{s}$ for a sufficiently high Reynolds number, as confirmed by (Loder and Hamilton 1991).

For the mooring system at hand, vortex shedding causes oscillating drag, lift, and side force components, which not only corrupt the measurements taken, but compromise the structural integrity of the system, leading to fatigue over time resulting from high-frequency vibration. To minimize these effects, a new streamlined buoy shape is proposed, designed, and tested to minimize drag. In addition, the oscillation dynamics of this buoy under conditions conducive to vortex shedding are investigated, with the goal of eliminating, or at least minimizing, the magnitude of the oscillations induced. 


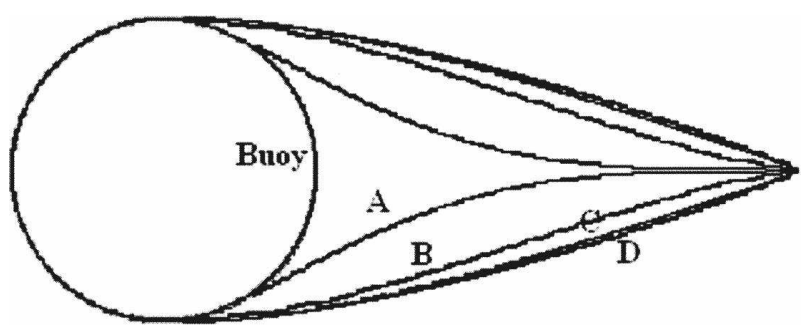

FIG. 1. Proposed rear-cowling shapes of the optimal ratio $t / c$; A: teardrop, B: pear shape, C: droplet, and D: NACA4 rear cowling.

\section{Proposed cowling shapes}

We tested our approach on a sphere of the size of that commonly used-one with a diameter of $0.7 \mathrm{~m}$ and a net buoyancy of $900 \mathrm{~N}$. We propose the use of a streamlined cowling encapsulating the original buoy. For each cowling, the attachment point (swivel axis) of the mooring line is at the widest point of the enclosed spherical buoy. Buoys are typically constrained to swivel in the horizontal plane only, and hence the alterations to the buoy design by way of the addition of cowlings are assumed to minimize drag-induced motion resulting from fluid flow in the horizontal orientation. We ignore vertical motion as being much smaller than the horizontal velocity, which is a reasonable assumption for most conditions.

We defined, parametrically, four families of basic "teardrop" shapes [teardrop (Van Seggern 1993), pear shaped droplet (Van Seggern 1993), and NACA4 (National Advisory for Aeronautics)] and separated them into two classifications of cowlings (rear or full). "Rear" cowlings are attached only to the rear of the sphere, and for "full" cowlings the sphere is fully enclosed by the cowling.

Because the drag coefficients of rotationally symmetric bodies depend to a high degree upon the ratio of the maximum diameter to length $t / c$, from a first-order perspective it is useful to choose the parameters for each of the cowling shapes by minimizing the drag coefficient as related to this ratio. Based upon the maximum frontal area, the dependence of the pressure drag coefficient is related to $t / c$ by (Hoerner 1965)

$$
C_{D \text { front }}=C_{f}\left[3(c / t)+4.5(t / c)^{1 / 2}+21(t / c)^{2}\right],
$$

a relation that is minimized when $t / c$ is 0.39 .

Using the optimal ratio, we obtain several different cowling shapes for the buoy exposed at the leading edge (Fig. 1) and others with the buoy completely surrounded by cowlings at each end of the flow (Fig. 2). It bears noting that these basic shapes used in the cowlings differ most notably in the existence and location of a point of inflection. The teardrop-shaped cowling has

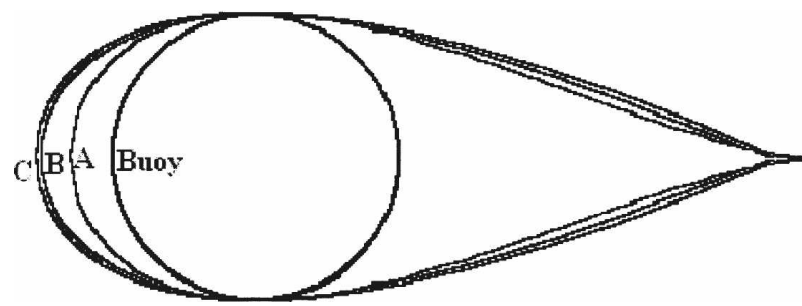

FIG. 2. Proposed full-cowling shapes for the optimal ratio $t / c$; A: pear shape, B: droplet, and C: NACA4 full cowling.

a inflection point located at about one-third of the total cowling length, whereas the pear-shaped cowling has one located at about two-thirds of its total cowling length measured aft of the nose The droplet- and NACA 4-shaped cowlings have no inflection points.

Due to concerns that, from a practical standpoint, the optimal shapes not be too lengthy and cumbersome, a shortened version of the droplet-shaped full cowling was chosen with a ratio $t / c$ of 0.60 : the "truncated droplet."

\section{Computational fluid dynamic analysis of proposed buoys}

A CFD software package (Fluent) was used to simulate the flow about the various buoy shapes and to estimate the lift, drag, and yawing moment coefficients thereof. Fluent uses an unstructured, finite-volumebased solver to predict the fluid dynamic properties of a computational model. To begin the calculations, a mesh is grown about the geometrical representation of the buoy and entered into the fluid dynamical software. Multiple iterations of the Navier-Stokes equations then converge to the final simulation, which is set as a convergence limit of $0.1 \%$ by the Fluent software (Fluent 2005).

Fluent solves the governing integral equations for the conservation of mass and momentum, and (when appropriate) for energy and other scalars such as turbulence and chemical species. A control volume-based technique is used in which the domain is divided into discrete control volumes using a computational grid (the "mesh"). The governing equations are integrated on the individual control volumes to construct algebraic equations for the discrete dependent variables ("unknowns"), such as velocities, pressure, temperature, and conserved scalars. Finally, linearization of the discretized equations and solution of the resultant linear equation system produces updated values of the dependent variables (Fluent 2005).

In these calculations, the segregated solver of Fluent was used. Using this approach, the governing equations 

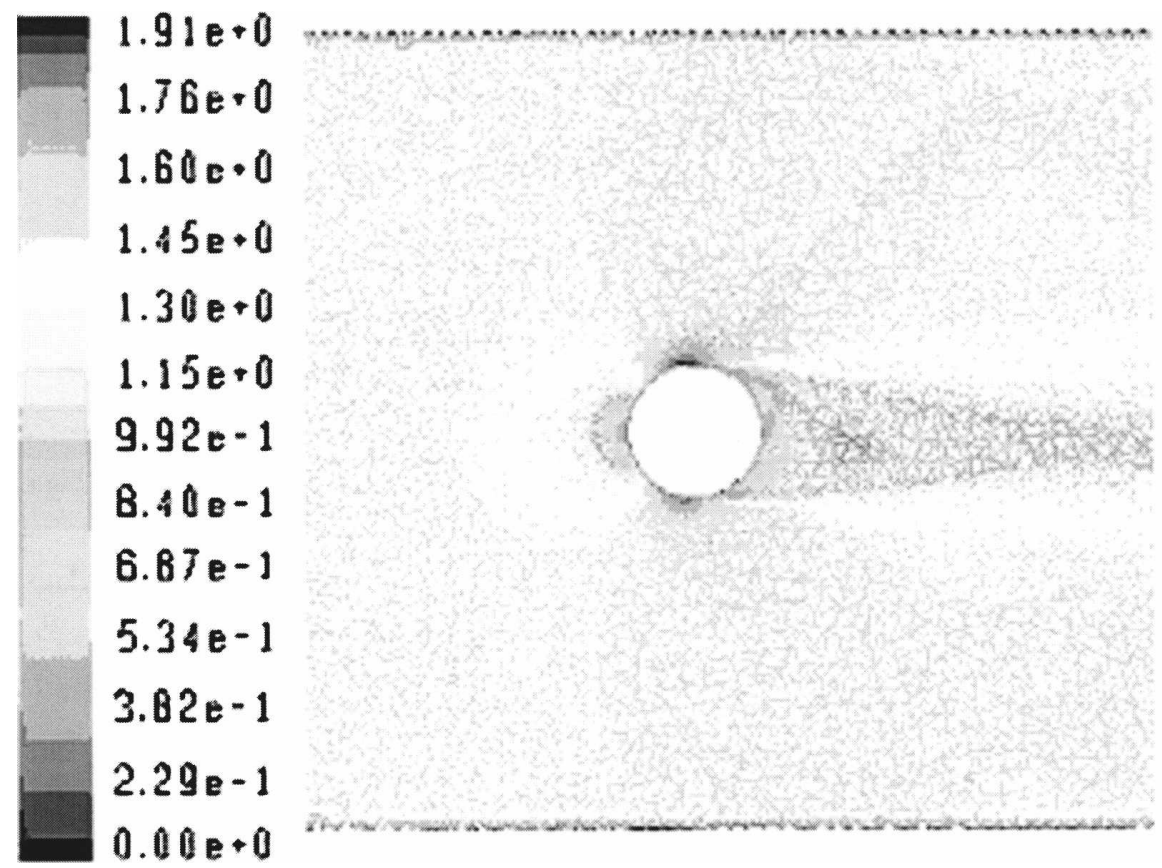

$1.600 \cdot 0$

$1.15 e+0$

$9.92 \mathrm{e}-1$

B. $40 \mathrm{e}-1$

$6.87 e^{-1}$

$5.34 \mathrm{e}-1$

$3.82 \mathrm{e}-1$

$2.29 p-1$

FIG. 3. Velocity contours $\left(\mathrm{m} \mathrm{s}^{-1}\right)$ of the steady-state estimation produced by CFD simulation.

are solved sequentially (i.e., segregated from one another). Because the governing equations are nonlinear (and coupled), several iterations of the solution loop must be performed before a convergence is reached. The resulting simulation is dependent upon the initial boundary conditions imposed and the grid size of the mesh, because flow conditions are calculated at each node in the mesh. For a sufficiently fine mesh, the results of the simulation may be deemed independent of the mesh size and growth. Meshes on the face of the buoy were less than $1 \%$ of the length of the buoy studied, and the meshes throughout the volume representing the fluid surrounding the buoy were $1 \%$ of the length of the rectangular fluid "pipe" used.

It is important that the pipe is large enough that the walls thereof do not affect the solutions. To this end, the buoy was located 1.5 buoy lengths downstream from the inlet of the fluid flow, five buoy lengths upstream from the outlet, and three buoy lengths from the pipe walls. As well, because of practical issues of construction and meshing, the extreme trailing edges of the optimally revolved shapes were truncated to a rounded shape. Such truncations do not significantly alter the length or shape of the optimal shapes discussed earlier.

Steady-state (time independent) solutions were obtained by iterating with the steady solver setting in Fluent to determine the average response of the proposed buoys. The second stage was to invoke the unsteady (time dependent) solver to accurately estimate the time evolution of the vortices and inherent oscillations expected in the forces and moments acting upon the buoy. Furthermore, all simulations were carried out for the zero angle of attack of the approach flow. This assumption is valid for buoys moored in the relatively sheltered Bonne Bay region. Here the waves are typically of amplitudes of less than $1.5 \mathrm{~m}$, and have periods of 5-6 s and wavelengths of about $30 \mathrm{~m}$.

To maintain flow conditions consistent with the fullscale prototype $\left(0.71-\mathrm{m}\right.$ diameter in uniform $1 \mathrm{~m} \mathrm{~s}^{-1}$ flow), we ran the CFD simulations with a freestreaming velocity of $0.71 \mathrm{~m} \mathrm{~s}^{-1}$ and a sphere diameter of $1 \mathrm{~m}$. The time step to ensure numerical stability was $0.01 \mathrm{~s}$.

\section{a. Steady-state analysis}

\section{1) Spherical Buoy}

As can be seen (Fig. 3), there is a considerable wake aft of the sphere at a Reynolds number of about $10^{6}$, which produces fairly large drag forces. Indeed, Fluent reports a drag coefficient of 0.55 based on a maximum frontal area, a value that is in good agreement with conventional experimental results (Berteaux 1991).

\section{2) Spherical BuOy With attached COWLings}

The cowlings dramatically reduce the drag force experienced, reducing it to about $25 \%$ of that of the 


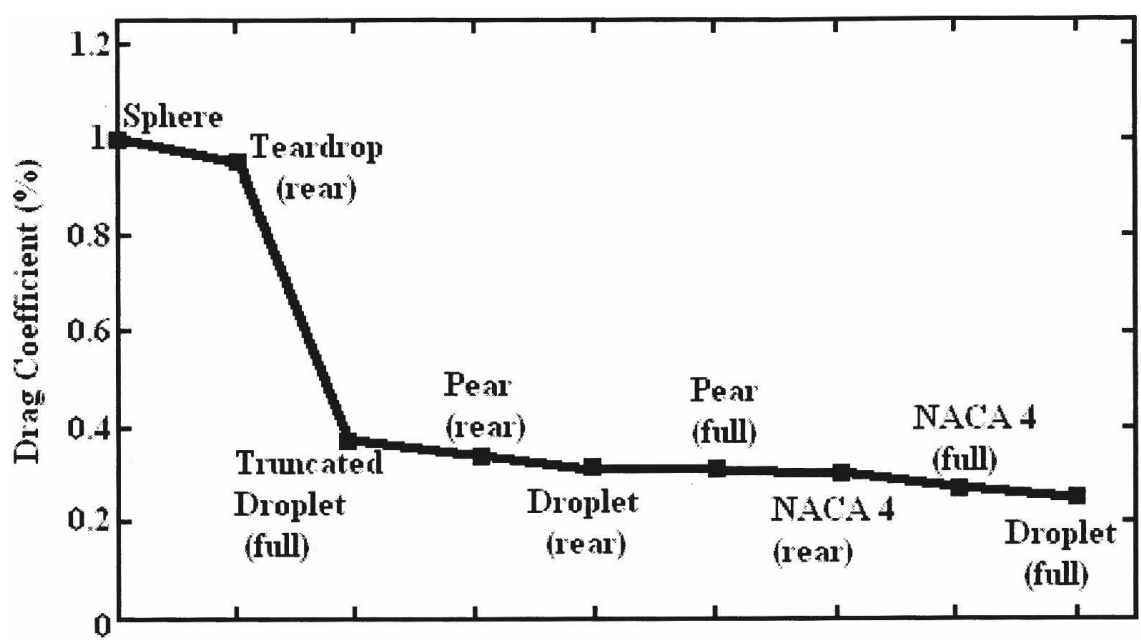

FIG. 4. Summary of drag coefficient results for steady-state analysis.

sphere. Furthermore, the wake aft of the concatenated spherical buoy and cowlings is much narrower than that of the original spherical buoy, which implies that the effects of vortex shedding should be reduced.

It is interesting to note (Fig. 4) that the existence and location of a point of inflection does seem to affect the drag force acting upon the buoys. Taking the rear cowlings as examples, the calculated drag coefficients increase with increasingly forward locations of inflection points, which physically makes sense because the points of inflection indicate regions that are in a sense not as "smooth" and "continuous" as those regions with uninterrupted concavity. It is natural to expect the boundary layers to experience increased disturbance from such "discontinuities" because the appearance of an adverse pressure gradient is usually associated with the presence of a point of inflection in the shape of a surface.

Considering all of the cowling shapes presented here, the droplet-shaped full cowling minimized the drag and the downstream excursion of the buoy and the effect of mooring excursion on scientific sensors mounted on the line (Fig. 5). As well, the reduced wake width implies a reduction in vortex-induced vibrations, which can corrupt the signals from the sensors as well as be structurally damaging over time.

A submerged body is considered to be stable if the center of effort of the body occurs behind the swivel axis, and thus the body will self-align in flow (Hoerner and Borst 1975). The center of effort is the threedimensional analog of the aerodynamic center for a two-dimensional body. The center of effort thus indicates the location on the longitudinal centerline of the body of a transverse axis about which the net hydrodynamic moment would be zero. For axisymmetric bodies the moment is typically reported about a transverse axis through the center of buoyancy denoted by $C_{b}$. When the net moment is to be reported about an axis that is a distance $x_{\mathrm{CE}}$ ahead of the $C_{b}$, that moment is given by

$$
M_{\text {yaw }}\left(x_{\mathrm{CE}}\right)=M_{\text {yaw }}\left(C_{b}\right)-F_{\text {side }} x_{\mathrm{CE}},
$$

where $F_{\text {side }}$ is the measured hydrodynamic side force and $M_{\text {yaw }}$ is the yawing moment acting on the body. From this moment-transfer relation, if the net hydrodynamic moment is to be zero, the transverse axis must be located at a distance $x_{\mathrm{CE}}$ ahead of the $C_{b}$, and the location of the center of effort $C_{e}$ is given by

$$
x_{\mathrm{CE}}=M_{\text {yaw }}\left(C_{b}\right) / F_{\text {side }} .
$$

Much study has been carried out on two-dimensional bodies; however, the behavior of three-dimensional streamlined bodies is relatively unknown in terms of the center of effort. The location of the center of effort of such bodies has obvious implications in the present study because it is desired that the final buoy shape be self-aligning. For the droplet-shaped full cowling, the center of effort is located aft of the point of attachment to the mooring line (and hence the axis of swivel), so, as is, the buoy is self-aligning and stable.

\section{b. Unsteady-state simulations}

Unsteady simulations were run for the most promising cowling shape (droplet) as well as for the original spherical buoy to see the effect of vortex shedding upon the forces acting upon the buoy, and to confirm the implied reduction of the vortex shedding by the more streamlined shape.

The drag, lift, and transverse forces acting upon a bluff body resulting from vortex shedding should oscil- 


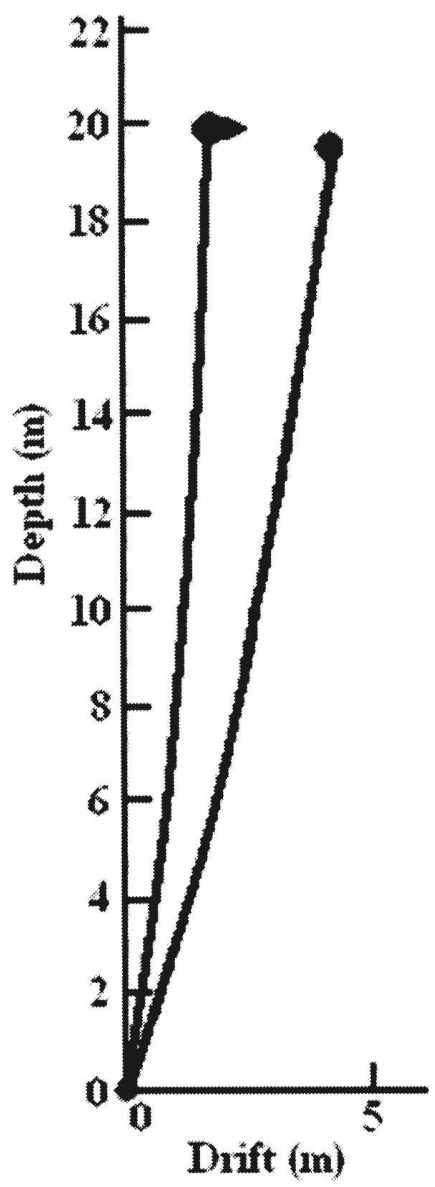

FIG. 5. Excursions from the vertical experienced by the original spherical buoy and the droplet-shaped full cowling, given a uniform current of $v=1 \mathrm{~m} \mathrm{~s}^{-1}$.

late coherently in a manner consistent with the vortices that are shed. Because the vortices are shed at a frequency of $f_{s}=S(v / d)$, the drag, lift, and transverse forces are all expected to oscillate close to $f_{s}$ for a truly three-dimensional wake, such as those of the proposed and tested buoys.

Again, we consider a unit-diameter spherical buoy in a net horizontal flow field of speed $0.71 \mathrm{~m} \mathrm{~s}^{-1}$, implying that $f_{s} \sim 0.154 \mathrm{~Hz}$ when the Strouhal number has a value 0.22 . Thus, we would expect the drag, lift, and transverse forces to oscillate at $0.154 \mathrm{~Hz}$. Because the proposed buoys with the cowling attachments also have the same effective characteristic dimension, we expect the frequencies of the vortex shedding, and hence of the oscillating forces, to be the same as those calculated for the original spherical buoy, with much-reduced amplitudes of vibration.

Using the same mesh as designed for the steady calculations, unsteady simulations were run for the original spherical buoy and the droplet-shaped full cowling
TABLE 1. Summary of drag and lift coefficients for numerical calculations.

\begin{tabular}{lcc}
\hline \hline & $\begin{array}{c}\text { Steady-state drag } \\
C_{D} \text { and lift } C_{L}\end{array}$ & $\begin{array}{c}\text { Unsteady-state } \\
\text { (mean values) drag } \\
C_{D} \text { and lift } C_{L}\end{array}$ \\
\hline Sphere & $0.55-0.09$ & $0.68-0.12$ \\
Droplet & $0.14-0.014$ & $0.08-0.001$ \\
\hline
\end{tabular}

using the same flow parameters and a time step of 0.01 s. From careful analysis of the resulting force data, the effects of the vortex action are easily observed. For both of these axisymmetric shapes, the drag and lift forces oscillate at $f_{s} / 2$, which is different from what is observed for two-dimensional shapes. The average drag and lift coefficients thus obtained agree well in magnitude with those reported from the steady-state simulations, with small discrepancies that are to be expected from such numerical methods, as highlighted in Table 1.

It should be noted that the lift calculated for the sphere is nonzero; however, experimentally it is known that a sphere has zero lift. The discrepancy here is most likely due to numerical error introduced by factors such as grid size and concentration. In any case, the lift force thus determined is about one-sixth that of the drag force and thus is not a major concern.

The oscillations in the drag coefficient are much smaller in amplitude than those observed for the sphere, as well as those observed in the lift coefficient of the same simulation (Figs. 6 and 7). However, it is important to note that the frequency of the vortex shedding appears to be about the same, so the simulations seem to be self-consistent. While the drag and lift coefficients of the droplet-shaped full cowling oscillate significantly, these oscillations occur at very low amplitudes in comparison to those resulting from shedding from the original spherical buoy. In fact, when the results from both simulations are plotted together (see Figs. 6 and 7), the oscillations observed in the dropletshaped full cowling become negligible because the forces appear to be nearly constant in comparison to those of the sphere. The strength of the vortices is proportional to the square of the average amplitude of the force fluctuations; the vortices shedding from the droplet-shaped full cowling are only $0.3 \%$ as strong as those shedding from the spherical buoy.

\section{Scaled model water tunnel experiments}

Scaled model experiments of the sphere, the droplet, and the truncated droplet were carried out to confirm the results obtained from the computational fluid dynamic analysis. The scaled models were constructed 


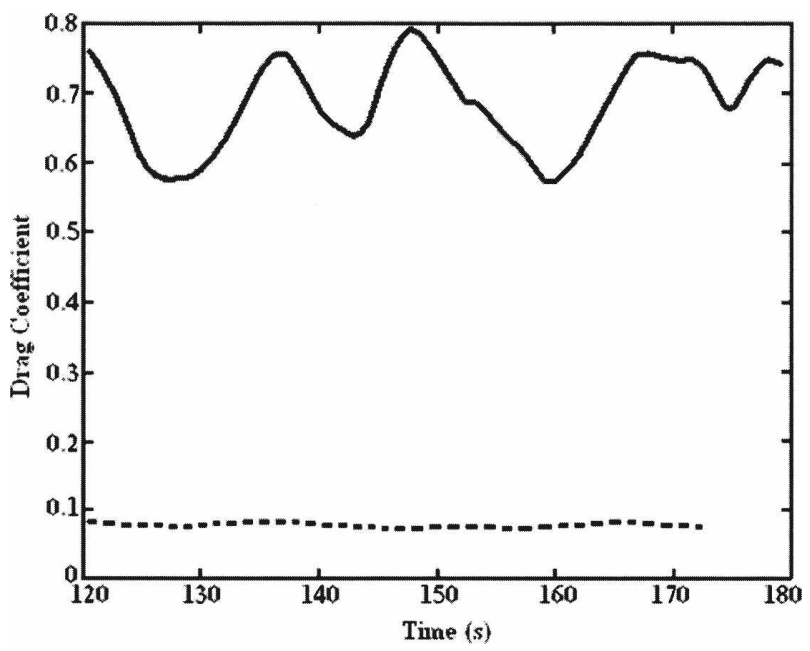

FIG. 6. Comparison of drag coefficient for the spherical buoy (solid) and the droplet-shaped full cowling (dashed).

with a maximum diameter of about $10 \mathrm{~cm}$, roughly $1 / 10$ of the prototype dimension. The scaled model of the sphere was constructed of Huntsman RenShape, while the droplet and truncated droplet models were fabricated of polished aluminum. All three models were then clear coated to ensure consistent surface texture. As well, matching struts were constructed for each of the three scaled models to suspend and anchor the models in the cavitation tunnel (Fig. 8). The dimensions of the three scaled models are given in Table 2 .

Capable of maximum water speeds of about 10 $\mathrm{m} \mathrm{s}^{-1}$, depending upon the degree of tunnel blockage, the cavitation tunnel consists of a circulating water column and testing cross section of $2500 \mathrm{~cm}^{2}$ and is equipped with a six-component balance, enabling mea-

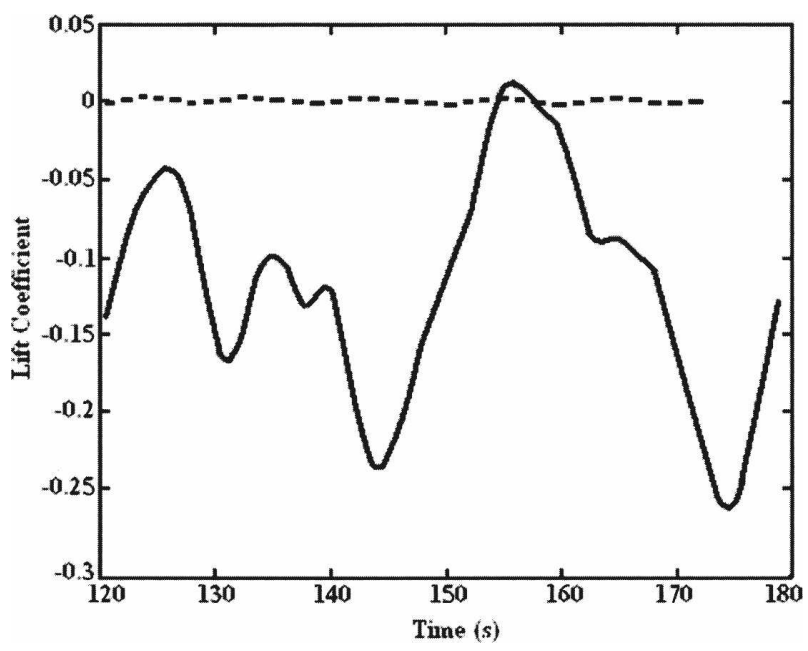

FIG. 7. Comparison of lift coefficient for the spherical buoy (solid) and the droplet-shaped full cowling (dashed). surements of three orthogonal forces and the corresponding moments. This balance was calibrated, prior to these experiments, by hanging known masses in each of the three orthogonal axes.

We collected data from the six-component balance using a data acquisition system with a sample rate of $100 \mathrm{~Hz}$ for each signal. Each model was anchored to the underside of the balance (which is flush with the ceiling of the test section) by its corresponding strut, and the water was circulated from about 0.5 to roughly $9 \mathrm{~m} \mathrm{~s}^{-1}$. Correspondingly, each strut was run alone through the same range of water speeds so that, by subtraction, the effects and forces experienced by the model alone could be obtained. Furthermore, for the droplet and truncated droplet models, this procedure was repeated at varying small angles of yaw so that the location of the center of effort $\left(C_{e}\right)$ for each shape could be calculated.

\section{a. Steady-state analysis}

\section{1) SPHERICAL SCALED MODEL}

The time-averaged drag force on the sphere depends quadratically on water speed (Fig. 9). Converting this force data into drag coefficients based upon maximum frontal area, the drag coefficient approaches the accepted value of 0.5 , and hence the value obtained by the computational fluid dynamic analysis of 0.55 is substantiated. Using the side forces, the lift force is recorded as being near zero for all flow speeds, at a magnitude of about $1 \%$ of the drag force.

\section{2) Droplet-Shaped SCALED MODEL}

The droplet-shaped full-cowling model was made of solid aluminum, which was then polished and coated with a clear lacquer. This model has a maximum diameter of $9.95 \mathrm{~cm}$, an overall length of $28.9 \mathrm{~cm}$, a swivel axis at a distance of $4.98 \mathrm{~cm}$ aft of the nose, and a $C_{b}$ at a distance of $11.89 \mathrm{~cm}$ aft of the nose.

The drag coefficient approaches a value of 0.08 , which is well in keeping with the CFD estimate of 0.08 . Furthermore, the droplet model experiences a nonzero but minimal side force; however, the effects of the onset of turbulence exist as before in a finite range of flow speeds.

Using the time average of the measured side forces and the measured yawing moment about an axis through the center of buoyancy, one can obtain the location of the center of effort of the droplet model by taking the limit to zero of the ratio of the yawing moment to the net side force. Hence, the center of effort of the droplet model occurs about $0.30 \mathrm{~m}$ behind the center of buoyancy and since the center of buoyancy itself 


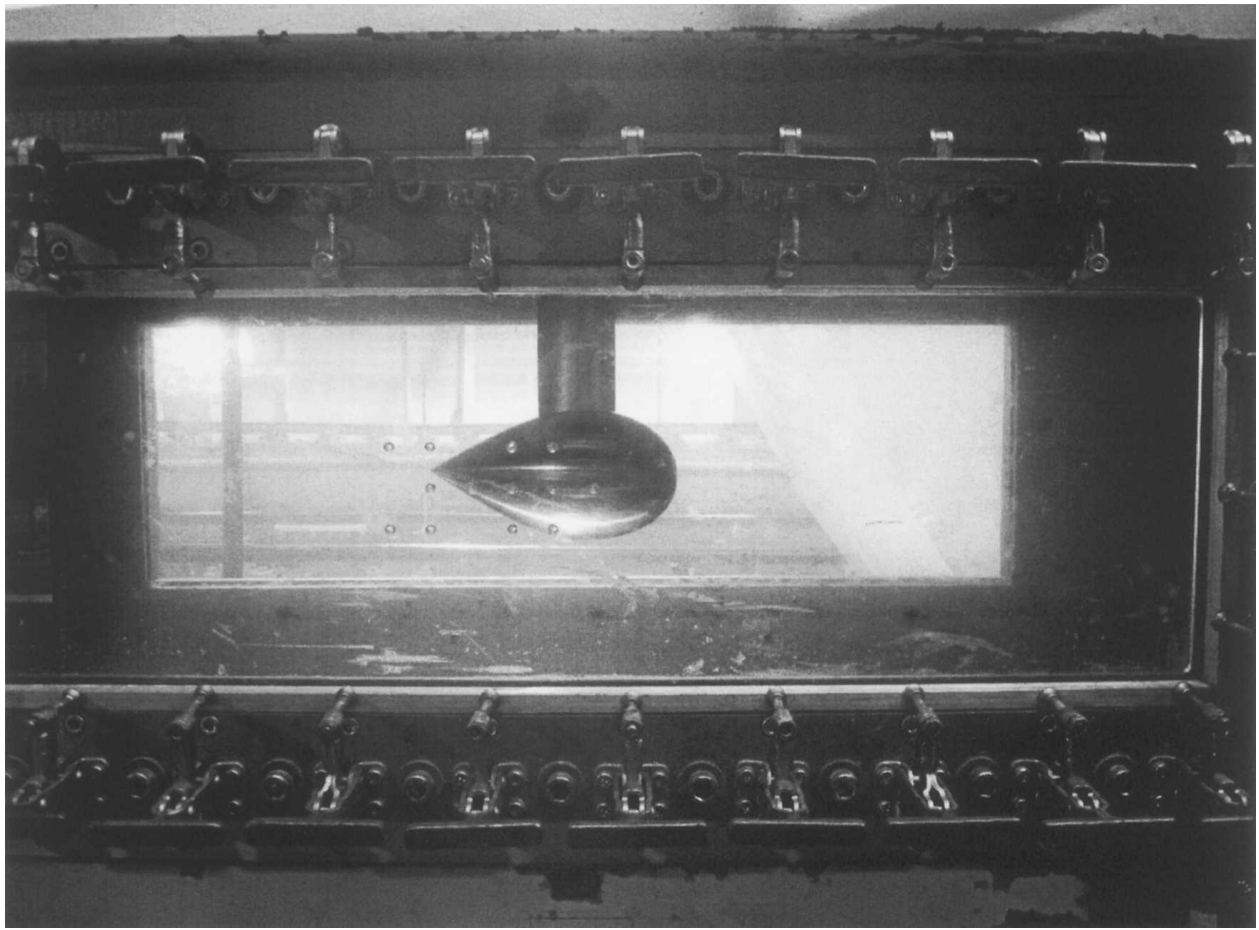

FIG. 8. Cavitation tunnel viewing panel with model in place, at the NRC Canada's Institute for Ocean Technology, St. John's, Newfoundland, Canada. The horizontal scale is roughly $1.5 \mathrm{~m}$.

is aft of the axis of swivel, the droplet model is selfaligning for all flow speeds tested, as previously suggested by the CFD results.

\section{3) TRUNCATED DROPLET-SHAPED SCALED MODEL}

This model has a maximum diameter of $9.95 \mathrm{~cm}$, an overall length of $16.6 \mathrm{~cm}$, a swivel axis at a distance of $4.81 \mathrm{~cm}$ aft of the nose, and a $C_{b}$ at a distance of $6.82 \mathrm{~cm}$ aft of the nose.

As expected, due to the shortened stature of the truncated droplet, the drag force experienced is slightly higher than that of the full-length droplet. Converting the obtained drag force data into a drag coefficient based upon maximum frontal area, the drag coefficient approaches a value of 0.14 close to the CFD estimate of 0.19 . The truncated droplet model experiences a nonzero but minimal side force; however, the effects of the onset of turbulence exist as discussed before for a finite range of flow speeds.
Again using the measured time-averaged side forces and the yawing moment about an axis through the center of buoyancy, one can obtain the location of the center of effort of the truncated droplet model. The location of the center of buoyancy of the truncated droplet is just short of $1 \mathrm{~cm}$ aft of the center of the collocated sphere, but because the center of effort occurs about $0.09 \mathrm{~m}$ behind the center of buoyancy, the truncated droplet is self-aligning for all flow speeds tested.

\section{b. Unsteady-state analysis}

Because the characteristic dimension for all three scaled models is the same $(9.95-\mathrm{cm}$ maximum diameter), it is reasonable to expect that all three will experience a roughly equal frequency of vortex shedding. However, a result of streamlining for drag reduction is a reduction of the wake width, of which the immediate implication of interest is that the effects of vortex shed-

TABLE 2. Dimensions of the tested scaled models.

\begin{tabular}{|c|c|c|c|c|}
\hline Model & $\begin{array}{c}\text { Maximum } \\
\text { diameter }(\mathrm{cm})\end{array}$ & $\begin{array}{c}\text { Overall } \\
\text { length }(\mathrm{cm})\end{array}$ & $\begin{array}{l}\text { Distance from nose } \\
\text { to swivel axis }(\mathrm{cm})\end{array}$ & $\begin{array}{c}\text { Distance from nose } \\
\text { to } C_{b}(\mathrm{~cm})\end{array}$ \\
\hline Sphere & 9.95 & 9.95 & 4.975 & 4.975 \\
\hline Droplet & 9.95 & 28.91 & 8.38 & 11.89 \\
\hline Truncated droplet & 9.95 & 16.59 & 4.81 & 6.82 \\
\hline
\end{tabular}




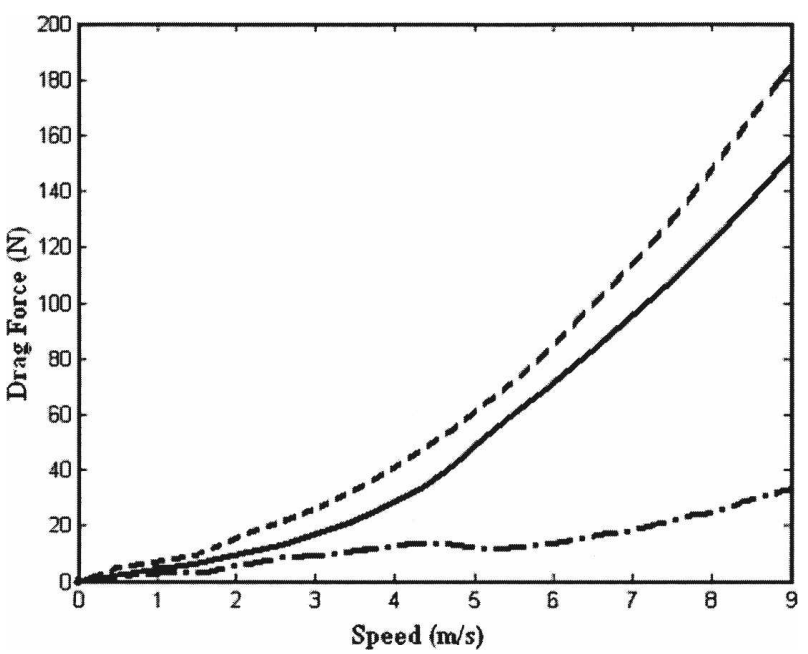

FIG. 9. Drag force (N) on the sphere as a function of flow speed: total drag force registered by the load cell (dashed line), the drag force recorded for the strut alone (dash-dot line), and the subtracted value representing the drag force due to the sphere alone (solid line).

ding are reduced. Hence, the lower drag shapes should experience less energetic vortex shedding. The Reynolds number and vortex-shedding frequency do not scale proportionally; while the relative power of the shed vortices can be compared with the relative results generated by the CFD analysis, no such comparison can be retrieved about the frequency of their shedding.

\section{1) SPHERICAL SCALED MODEL}

The data were analyzed for each of the four highest speed intervals recorded for the spherical scaled model, but because of excessive vibrations in the mounting system, the spherical scaled model was tested up to flow speeds of only about $6.25 \mathrm{~m} \mathrm{~s}^{-1}$. The power spectra show energy at frequencies from low frequencies up to about $10-15 \mathrm{~Hz}$, in some agreement with theory that predicts $f_{s}$ should vary linearly between 10 and $14 \mathrm{~Hz}$. As well, the root-mean-square amplitudes of the oscillating drag forces were determined to increase from 2.6 to $5.5 \mathrm{~N}$ a linear fashion with increasing flow speed; the corresponding root-mean-square values of the drag coefficient increased from 0.031 to 0.036 while the mean drag coefficient increased from 0.46 to 0.51 .

\section{2) Droplet-Shaped SCALED MODEL}

The power spectra for the droplet-shaped model showed high-frequency $(\sim 50 \mathrm{~Hz})$ oscillations associated with the tunnel motor revolutions and lowerfrequency oscillations (up to $15 \mathrm{~Hz}$ ) attributable to the droplet and strut. At the high limiting speeds tested, between 7.82 and $9.38 \mathrm{~m} \mathrm{~s}^{-1}$, theory predicts that $f_{s}$ should range linearly between 17.3 and $20.1 \mathrm{~Hz}$. Because experimentally the drag force power spectra have energy up to $10-15 \mathrm{~Hz}$, generally the frequency of vortex shedding observed experimentally is slightly lower than that predicted theoretically. The root-meansquare amplitudes of the oscillating drag forces ranged from 0.13 to $1.1 \mathrm{~N}$, increasing with flow speed; the corresponding root-mean-square values of the drag coefficient increased from 0.00055 to 0.0032 . Vortex energy was much less for the droplet shape than for the sphere or the truncated droplet.

\section{3) TRuncated DRoplet-SHAPEd SCALED MOdEL}

The power spectra for the truncated droplet again showed high-frequency $(50 \mathrm{~Hz})$ oscillations associated with the tunnel motor revolutions and lower-frequency oscillations (up to $15 \mathrm{~Hz}$ ) attributed to the truncated droplet and strut. At speeds between 7.82 and 9.39 $\mathrm{m} \mathrm{s}^{-1}$, theory predicts that $f_{s}$ should range linearly between 17.3 and $20.1 \mathrm{~Hz}$. Experimentally, strongly redshifted energy spectra are observed, with little energy detected above $10 \mathrm{~Hz}$, well below the predicted range of frequencies. As well, the root-mean-square amplitudes of the oscillating drag forces were determined to range from 2.5 to $3.0 \mathrm{~N}$, increasing with flow speed; the corresponding root-mean-square values of the drag coefficient decreased from 0.011 to 0.0088 .

\section{c. Comparison of scale models}

Unfortunately, vibrations in the cavitation tunnel limited the extent to which the spherical model could be safely tested; however, by extrapolation, we can estimate the behavior at slightly higher flow speeds such as would be experienced by the prototype. We have no laboratory data to support these extrapolations, but they are probably reasonable estimates of the maximum likely drag. Extrapolation of the root-meansquare amplitude of the force fluctuations acting on the sphere at higher speeds, matching the maximal speeds attained by the proposed cowling shapes as well as the speeds used in the numerical comparative study (Fig. 10), does indeed highlight that the amplitude of the force fluctuations resulting from the shedding of vortices is greatly reduced by the application of drag- and hence wake-reducing cowlings. Because the power of the shed vortices is proportional to the square of the root-mean-square amplitudes of the measured forces, the addition of such cowlings well reduces the effect of the shed vortices because the root-mean-square amplitudes are only a fraction of that of the sphere. 


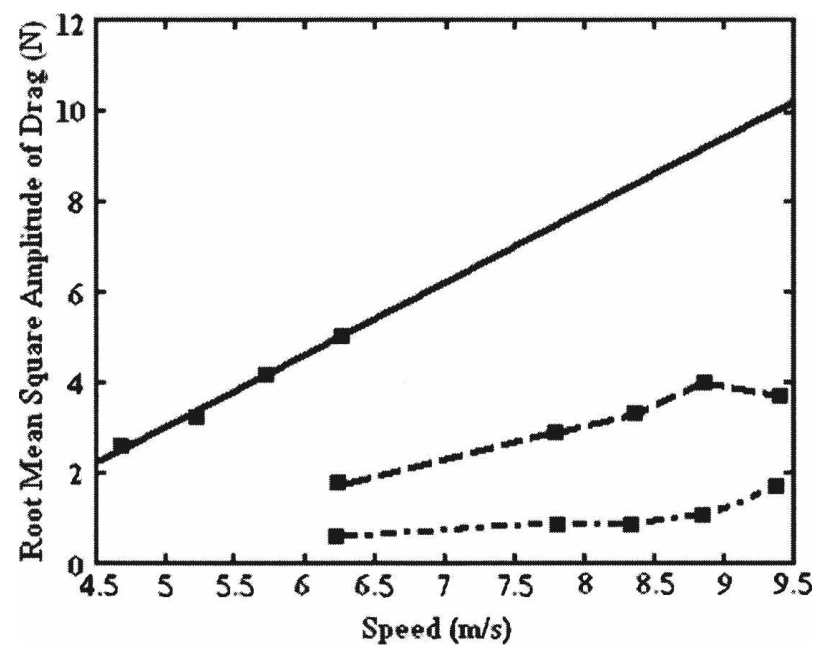

FIG. 10. Comparison of root-mean-square amplitudes of drag force oscillations of the sphere- (solid), the droplet- (dash-dot line), and the truncated droplet- (dashed) shaped scaled models.

\section{Discussion and conclusions}

Beginning with a spherically shaped buoy of $0.71-\mathrm{m}$ diameter and $900-\mathrm{N}$ net buoyancy, the primary goal was to reduce the mean drag force and the effects of vortex shedding caused by the impingent current on the bluff buoy. This goal was achieved by (a) computational fluid dynamic analysis of seven streamlined shapes, and confirmed by (b) scaled model experiments in a water tunnel on two of the most promising shapes. For the fullscale mooring, for each cowling, the attachment point (swivel axis) of the mooring line is at the widest point of the enclosed spherical buoy, not at the center of buoyancy of either a water-tight or free-flooding cowling.

The full cowlings (which totally enclose the existing spherical buoy) prove to be the most efficient at reducing the drag force acting upon the buoy, yielding drag coefficients of less than $30 \%$ that of the original spherical buoy. In particular, from the CFD analysis completed, the droplet-shaped full cowling performs especially well, with a drag coefficient $19 \%$ that of the spherical buoy and a center of effort well behind the swivel axis, thus rendering the resulting buoy to be selfaligning and stable. From a standpoint of practicality, a truncated droplet-shaped full cowling was designed and tested, resulting in a drag coefficient of $37 \%$ that of the spherical buoy and a center of effort occurring well behind the swivel axis. Both of these shapes reported finite, but negligible, time-averaged lateral force.

A secondary result of drag reduction is that the wake immediately aft of the buoy is greatly reduced in width, implying that the formation of vortices and shedding there are also reduced. Using time-dependent CFD

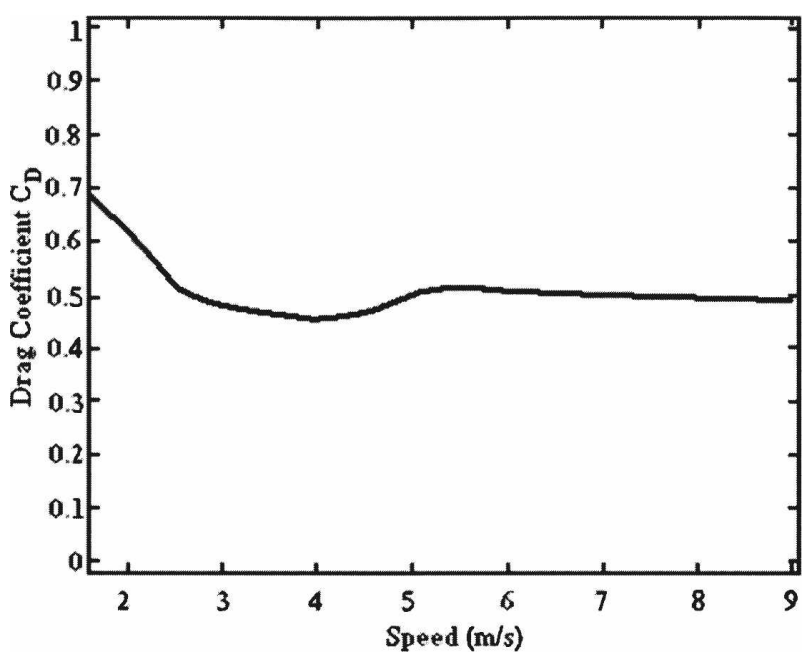

FIG. 11. Dependence of experimental drag coefficient for a sphere upon flow speed.

analysis, the presence and evolution of the shed vortices were also examined. The vortices shed aft of the spherical buoy are quite strong and the recorded orthogonal forces oscillate steadily at frequencies close to those expected. While the forces acting upon the proposed droplet-shaped buoy resulting from the shedding of the vortices also oscillate at nearly the same frequencies, these oscillations occur at very low amplitudes in comparison to those for the original spherical buoy. In fact, the strength of the vortices shed from the dropletshaped full cowling is only $0.3 \%$ that of those shed from the spherical buoy, so the streamlining appears to have substantially reduced the action of the produced vortices on the buoy system.

Physical scaled model experiments were conducted in a cavitation tunnel with a scaled model sphere, full droplet-shaped solid cowling, and truncated dropletshaped solid cowling; the measured forces and moment confirmed the CFD analysis, mirroring the predictions quite well.

Both numerical experiments and scaled model water tunnel experiments show that the proposed cowlings dramatically reduced the drag force experienced on the original spherical buoy. Solid scaled models of the sphere, the droplet-shaped full cowling, and the truncated droplet-shaped full cowling were tested, indicating that at a Reynolds number of about $10^{5}$, the dropletshaped full cowling reduced the drag coefficient from 0.5 to 0.08 and the truncated droplet-shaped full cowling reduced it to 0.18 . As expected, the drag coefficients do depend upon flow speed (Fig. 11).

The results of the scaled model tests agreed well with the previous numerical predications computed by CFD analysis, within a differential of several percent 
TABLE 3. Comparison of drag coefficients generated by CFD calculations and by model experiments.

\begin{tabular}{lcc}
\hline \hline & $\begin{array}{c}\text { Percent of drag } \\
\text { of sphere (CFD) }\end{array}$ & $\begin{array}{c}\text { Percent of drag of } \\
\text { sphere (scaled } \\
\text { model) }\end{array}$ \\
\hline Droplet & $18 \%$ & $16 \%$ \\
Truncated droplet & $37 \%$ & $35 \%$ \\
\hline
\end{tabular}

(Table 3). The drag coefficient of the sphere for the numerical and experimental results themselves agree quite well, within $10 \%$, where $C_{D \mathrm{CFD}}=0.55$ and $C_{D \mathrm{EXP}}=$ 0.50 .

Analysis of the unsteady properties of the scaled model system confirmed the theoretical result of the vortex-shedding frequency, and indeed because the characteristic dimension of all three scaled models is the same, the frequency of shed vortices is nearly equal for all three models.

We calculated the root-mean-square amplitudes for the spheres and the truncated droplet to estimate the effect of the shape on the vortex-induced oscillations. The droplet shape reduced the root-mean-square amplitude of the vortex-induced oscillations to $12 \%$ of that of the sphere, while the truncated droplet reduced it to $35 \%$ that of the sphere. Because the relative power of the shedding vortices is proportional to the square of the root-mean-square amplitude of the force oscillations, the proposed cowling shapes do effectively reduce (to $1.5 \%$ for the droplet and to $12 \%$ for the truncated droplet) the strength of the vortex shedding compared to that of a sphere.

Proposed future work includes the design, fabrication, and testing of a full-scale prototype of the fully enclosing "truncated droplet" shape. Initial testing will take place in the 200-m towing tank at the National Research Council (NRC) Institute for Ocean Technology; the drag force and lateral accelerations will be measured and the results will be used to confirm the results obtained in the present investigation. At-sea experiments will be performed using lateral accelerometers to determine whether unsteady lateral forces and moments are present, and current meters and video recordings will be used to determine the mean offset resulting from the drag force.

Acknowledgments. Support for this work was provided by the Natural Sciences and Engineering Research Council as a grant to BdeY and in the form of a scholarship to JH.

\section{REFERENCES}

Berteaux, H. O., 1991: Coastal and Oceanic Buoy Engineering. 2nd ed. Henri O. Berteaux, 285 pp.

Brucker, C., 2001: Spatio-temporal reconstruction of vortex dynamics in axisymmetric wakes. J. Fluids Structures, 15, 543554.

deYoung, B., K. M. Brown, R. S. Adams, and S. D. McLean, 2005: Design and deployment of the Bonne Bay Ocean Observatory $\left(\mathrm{B}_{2} \mathrm{O}\right)$. Proc. Oceans 2005, Vol. 1m, Washington, DC, MTS/IEEE, 855-860.

Fluent, Inc., cited 2005: FLUENT user's guide version 6.2. [Available online at http://202.41.85.84/doc/fluent6.2/help/html/ug// main_pre.htm.]

Hamilton, J. M., 2000: Vibration-based technique for measuring the elastic properties of ropes and the added mass of submerged objects. J. Atmos. Oceanic Technol., 17, 688-697.

— G. A. Fowler, and D. J. Belliveau, 1997: Mooring vibration as a source of current meter error and its correction. $J$. Atmos. Oceanic Technol., 14, 644-655.

Hoerner, S. F., 1965: Fluid Dynamic Drag: Practical Information on Aerodynamic Drag and Hydrodynamic Resistence. Hoerner, $259 \mathrm{pp}$.

— mation on Aerodynamic and Hydrodynamic Lift. Hoerner, $505 \mathrm{pp}$.

Loder, J. W., and J. M. Hamilton, 1991: Degradation of some mechanical current meters by high-frequency mooring or wave motion. IEEE J. Oceanic Eng., 16, 343-349.

McGothlin, J. C., 1982: Drag coefficients of long flexible cylinders subject to vortex induced oscillations. M.S. thesis, Dept. of Ocean Engineering, MIT, 86 pp.

van Seggern, D. H., 1993: CRC Standard Curves and Surfaces with Mathematica. CRC Press, 556 pp. 